Erschienen in: Engelberg, Stefan/Holler, Anke/Proost, Kristel (Hrsg.): Sprachliches Wissen zwischen Lexikon und Grammatik. - Berlin, Boston: de

Gruyter, 2011. S. 459-481. (Institut für Deutsche Sprache. Jahrbuch 2011), https://doi.org/10.1515/9783110262339.459

Christiane Fellbaum

\title{
Klassifikation des Verblexikons in WordNet und Abgleichung mit FrameNet ${ }^{1}$
}

\begin{abstract}
WordNet und FrameNet sind zwei umfangreiche lexikalische Ressourcen. Obwohl sie auf unterschiedlichen Theorien der lexikalischen Organisation basieren und die semantischen und syntaktischen Eigenschaften von Verben in beiden Ressourcen unterschiedlich dargestellt werden, wird mit beiden das Ziel verfolgt, die Regelmäßigkeit des Lexikons hervorzuheben. Seit Levin (1993) ist bekannt, dass die Selektion und Projektion von Argumenten eng mit den semantischen Eigenschaften von Verben zusammenhängt, und dass eine syntaktisch basierte Klassifikation des Verblexikons semantisch homogene Klassen ergibt. In diesem Beitrag werden die unterschiedlichen Ansätze von WordNet und FrameNet, das Verblexikon einheitlich zu klassiffzieren, bewertet. Es wird ein laufender Versuch beschrieben, beide Ressourcen teilweise miteinander abzugleichen, indem ausgewählte Verben in einem großen Korpus zunächst manuell mit Bezug auf Einträge in WordNet und FrameNet annotiert werden. Anschließend werden verschiedene Ansätze zur automatischen Abgleichung, wie z.B. der des deutschen SALSA-Projekts, im Überblick dargestellt.
\end{abstract}

\section{Einleitung}

Es besteht allgemeine Übereinstimmung darin, dass der lexikalische Engpass in der maschinellen Sprachverarbeitung durch bessere lexikalische Ressourcen behoben werden kann und muss. Aber was sind ,bessere" Ressourcen? Eine mögliche Antwort setzt voraus, dass lexikalische Ressourcen sich auf unser Verstehen von menschlichem Sprachwissen stützen und dieses für maschinelle Anwendungen kodieren sollen. Manuell konstruierte Ressourcen basieren auf Introspektion und expliziten Regeln, die oft im Rahmen bestimmter, von diesen Ressourcen unabhängigen Theorien formuliert worden sind. Dies kann leicht zu inkompatiblen Analysen und Bedeutungsinventaren führen. Automatisch erstellte Ressourcen (vgl. Pantel/Lin 2002; Schulte im Walde ersch.), die auf Korpusextraktion basieren und nicht theoretisch vorbelastet sind, bringen zwar oft Regeln und Muster zum Vorschein, bergen aber die Gefahr in sich, unvollständig oder unausgewogen zu sein.

Die Arbeit wurde von der U.S. National Science Foundation (Grant IIS 0705155) unterstützt. Ich danke Collin Baker und Charles Fillmore für Kommentare zu einer früheren Version dieses Aufsatzes und den Bandherausgebern für ihr sorgfältiges Lektorat. 
Sprecherintuitionen zu Wortbedeutungen sind erstaunlich verschieden. Diskrepanzen zeigen sich deutlich in traditionellen Wörterbüchern sowie in modernen elektronischen Ressourcen, die den semantischen Raum, den ein Wort einnimmt, oft sehr unterschiedlich in zählbare und separate Lesarten aufteilen. Die Annotationen unterschiedlicher Annotierer, die die Tokens in einem Korpus mit entsprechenden Wörterbucheinträgen verknüpfen, stimmen oft nicht miteinander überein, da ein und dasselbe Token in einem bestimmten Kontext von unterschiedlichen Annotierern unterschiedlich interpretiert werden kann (vgl. Fellbaum/Grabowski/Landes 1997). Daher ist es nicht nur wichtig, verschiedene Ressourcen miteinander abzugleichen und somit eventuelle Ausreißer zu korrigieren, sondern auch die Ressourcen selbst mit einem Korpus abzugleichen. Im Folgenden wird zunächst über die Abgleichung von zwei, auf komplementären Modellen der lexikalischen Organisation basierenden Ressourcen, WordNet (Miller et al. 1990; Fellbaum (Hg.) 1998) und FrameNet (Baker/Fillmore/Cronin 2003), berichtet, die mittels der Annotation des American National Corpus (Ide/ Sudermann 2004) vorgenommen wird. Diese beiden Ressourcen werden schwerpunktmäßig im Hinblick auf die Struktur und die Alternationen der Argumente englischer Verben abgeglichen.

\section{WordNet}

WordNet ist eine lexikalische Datenbank in Form eines semantischen Netzes, die ursprünglich auf eine in den sechziger Jahren entwickelte und sowohl in der Psychologie als auch der Künstlichen Intelligenz gängige Theorie der Organisation des mentalen Lexikons gestützt war (Collins/Quillian 1969). Semantisch verwandte Wortformen sind durch lexikalische Relationen wie Synonymie, Antonymie und morphologische Ableitung miteinander verbunden. Kognitive Synonyme (im Sinne von Cruse 1986) sind in ungeordneten Mengen, so genannten Synsets, gruppiert. Die Lesarten polysemer Wortformen sind somit an die Zugehörigkeit zu verschiedenen Synsets gebunden; jede Kombination von Wortform und Synsetzugehörigkeit entspricht einer einmaligen Lesart (Miller et al. 1990; Fellbaum (Hg.) 1998). Zwei Lesarten des Verbs read sind z.B. in den zwei Synsets (1) und (2) (mit Beispielsätzen) dargestellt:

(1) learn, study, read, take („She is reading law at Oxford")

(2) read, register, show, record (,The gauge read empty ${ }^{c}$ )

Synsetzugehörigkeit in WordNet ist als Austauschbarkeit in vielen, aber nicht unbedingt allen Kontexten definiert. In vielen Kontexten ist eine bestimmte Wortform einer semantisch äquivalenten vorzuziehen: 
(3) The robbers tried to leave/\# exit the bank when the teller pressed the alarm button.

(4) Passengers are requested to exit/\#leave the bus through the rear door.

WordNets Verbsynsets sind nach rein semantischen Kriterien zusammengestellt. Folglich zeigen Verben, die in WordNet dem gleichen Synset zugeordnet sind, nicht immer dieselben syntaktischen Eigenschaften, wie in den Beispielen (5-7):

(5) My cats hate/detest/?loathe you.

(6) Lindsey hates/detests/loathes being known as a party girl.

(7) He will hate/??detest/??loathe to live in Milwaukee.

Auch Levins (1993) syntaktisch-basierte Klassifikation ordnet diese Verben ein und derselben Klasse (den Psych-Verben) zu; argumentstrukturelle Unterschiede wie die in den obigen Beispielen werden in Levins Klassifikation nicht berücksichtigt.

Synsets sind wiederum mittels konzeptuell-semantischer Relationen wie Hyperonymie, Meronymie und Kausalität verbunden; diese Relationen sind für alle Mitglieder eines Synsets identisch. Die meisten Relationen zwischen WordNets Verbsynsets basieren auf „Troponymie“, einer analogen Relation zu Hyperonymie im Nomenlexikon (Fellbaum 1990, 2002). Mehr oder weniger semantisch spezifische Verben werden mit Hilfe der Formel „X-en ist eine Art und Weise zu Y-en" in Baumstrukturen geordnet. Ein Beispiel ist der Baumausschnitt sich_fortbewegen, gehen, schleichen: schleichen ist eine Art zu gehen, gehen ist eine Art sich fortrubewegen, usw.

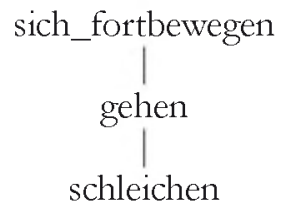

Die Zuordnung der Verben zu einem gegebenen Baum beruht auf der Intuition der Lexikografen, die sich dieser „Testformel“ ${ }^{\text {“ }}$ bedienen. Fellbaum (1990, 2002) zeigt, dass der Großteil des englischen Verblexikons so vernetzt werden kann; die für andere Sprachen entworfenen WordNets, einschließlich GermaNet (Kunze 2000), können die Troponymierelation ebenfalls als primäre Relation zur Verbverknüpfung anwenden. Neologismen sind prinzipiell Troponyme von schon etablierten Verben. Zum Beispiel kann das Verb e-mail einer Baumstruktur mit Kommunikationsverben als Troponym von urite zugeordnet werden. 
Schließlich zeigen die experimentell gewonnenen Daten von Fellbaum/ Chaffin (1990), Boyd-Graber et al. (2006) und Nikolova/Boyd-Graber/ Fellbaum (ersch.), dass Troponymie im mentalen Lexikon von Sprechern eine wichtige Rolle spielt. Als Relationen zwischen Verben und Verbsynsets kommen außerdem die folgenden Variationen von Entailment vor: „Cause" (give-have), „Proper Inclusion" (buy-pay), „Backward Presupposition" (succeed-try).

Fellbaum (1990) untersucht, ob sich aus der rein semantischen Organisation des Verblexikons in WordNet auch syntaktisch homogene Klassen ergeben. Dies ist oft, aber keineswegs immer, der Fall. Die semantische Unterklasse $\{g o$, travel, locomote $\}$ der Bewegungsverben bildet beispielsweise in syntaktischer Hinsicht eine homogene Klasse: Es sind alle intransitive Verben mit einer Präpositionalphrasenergänzung. In Bezug auf die Transitiv/ Intransitiv-Alternation verhält sich diese Klasse der Bewegungsverben aber uneinheitlich, vgl.:

(8) Kim walked/marched/promenaded her new dog this morning.

(9) *Kim strolled/ambled her new dog this morning.

In vielen Fällen zeigen die Verben in einer gegebenen Hierarchie unterschiedliche Argumentstrukturen. Ein bekanntes Beispiel ist die semantisch homogene Klasse der Transferverben, von denen nur eine Untermenge, die nicht semantisch, etymologisch oder morphologisch klar zu definieren ist, die Dativ-Alternation zeigt (Green 1974 inter alia):

(10) a. The professor gave an A to the best student.

b. Aunt Molly bequeathed her silver to Evelyne.

c. Mary donated $\$ 1,000$ to her favorite charity.

d. Peter conveyed his best wishes to the young couple.

(11) a. The professor gave the best student an A.

b. Aunt Molly bequeathed Evelyne her silver.

c. *Mary donated her favorite charity $\$ 1,000$.

d. *Peter conveyed the young couple his best wishes.

Die unterschiedlichen Argumentalternationen dieser intuitiv semantisch einheitlichen Verben sind in WordNet nicht berücksichtigt.

Umgekehrt gibt es Argumentalternationen, die scheinbar mit keiner semantisch kohärenten Verbklasse einhergehen. Die vielfach untersuchte Mittelkonstruktion (Keyser/Roeper 1984; Fagan 1992; Fellbaum/Zribi-Hertz 1990 inter alia) z.B. kann zwar grundsätzlich mit Verben, die eine Zustandsveränderung ausdrücken, auftreten (vgl. 12a-b und 13a-b), darüber hinaus erlauben aber auch manche Verben, deren Bedeutung keine Zustandsveränderung oder Affiziertheit involviert, und die keine eigenständige semantische Klasse bilden, diese Alternation (vgl. 12c-e und 13c-e): 
(12) a. The toddler broke the Chinese vase.

b. The tailor cut the silk.

c. My student sold his car in an hour.

d. The father photographed the triplets on Santa's lap.

e. The real estate agent showed us this attractive house.

(13) a. Chinese ceramics break easily.

b. The silk cut easily.

c. My student's car sold in an hour.

d. Triplets photograph nicely.

e. This attractive house shows nicely.

In manchen Fällen hängt die Verteilung von Argumentalternationen nicht nur von der Semantik der Verben, sondern auch von der der Argumente ab. Dies scheint bei der Instrument-Subjekt-Alternation der Fall zu sein, die bisher keine Erklärung gefunden hat (aber siehe Woicjil 1976), obwohl Sprecher robuste Intuitionen zur Akzeptabilität von Sätzen wie (14-15) zeigen:

(14) a. The key opened the door.

b. The knife cut the sausage.

c. The poison killed the rat.

(15) a. *The spoon stirred the soup.

b. *The pen signed the letter.

c. *The gun killed the prisoner.

Eine erste Konsequenz der hierarchischen Organisation von WordNet ist, dass manche Verben, die gemeinsame Argumentalternationen zeigen, wie z.B. die, die mit der Mittel- oder Instrument-Subjekt-Alternation vorkommen, verschiedenen Baumstrukturen zugewiesen sind.

Eine zweite Konsequenz von WordNets hierarchischer Organisation ist, dass verschiedene syntaktische Projektionen der Argumente eines Verbs als verschiedene Lesarten in separaten Bäumen erscheinen. Als Beispiel dienen hier die Verben, die die so genannte Lokativ-Alternation zeigen (Anderson 1971 inter alia). In (16) ist der Ort das direkte Objekt, und das Locatum wird als Präpositionalphrase realisiert. In (17) vertauschen diese beiden Argumente ihre syntaktischen Positionen:

(16) a. Load the truck with hay.

b. Spray the wall with paint.

(17) a. Load hay onto the truck.

b. Spray paint onto the wall.

Die Verben, die diese Argumentalternation zeigen, sind in WordNet je zwei verschiedenen Bäumen zugeordnet. Der Baum, der die Lesart in (16) repräsentiert, hat die Wurzel fill; die Lesart in (17) wird durch einen Baum mit der 
Wurzel place dargestellt. Alle zu letzterem Baum gehörigen Verben sind Troponyme von place. Obwohl die Beziehung zwischen diesen beiden Lesarten insofern systematisch ist, als sie eine große Verbklasse betrifft, ist sie in WordNet nicht kodiert. Das trifft auch auf andere Alternationsmuster wie etwa die Mittel-Konstruktion zu. Die Mittel-Lesarten in (13) (vgl. 13a: Chinese ceramics break easiby) sind alle einem Baum mit der Wurzel be (sein) zugeordnet, da sie eine Eigenschaft des strukturellen Subjekts ausdrücken; die transitiven Varianten in (12) (vgl. 12a: The toddler broke the Chinese vase) sind in einer Reihe von semantisch verschiedenen Bäumen vertreten.

Eine Ausnahme sind kausativ-inchoative Verben (break, move, grow usw.), deren transitiv-kausative und intransitiv-inchoative Lesarten (vgl. die Beispiele in (18) bzw. (19)) zwar in verschiedenen Bäumen repräsentiert, aber durch eine Cause-Relation verlinkt sind:

(18) a. The child broke the plate during dinner.

b. Farmers grow corn in New Jersey.

(19) a. The plate broke at dinner.

b. Corn grows in New Jersey.

Nicht alle Verben in einem gegebenen WordNet-Baum zeigen dasselbe syntaktische Verhalten. In manchen Fällen ist das syntaktische Potenzial bei den semantisch spezifischeren „Blätterknoten“ stärker eingeschränkt als bei den übergeordneten Verben; in anderen Fällen sind die durch solche Knoten repräsentierten Verben syntaktisch flexibler als die Wurzelverben; eine generell gültige Korrelation zwischen semantischer Spezifizität und Argumentalternationen scheint es nicht zu geben.

Zusammenfassend kann festgestellt werden, dass einerseits eine auf Argumentalternationen basierende Organisation des Verblexikons wie in Levin (1993) nicht immer semantisch homogene Klassen ergibt; umgekehrt ergibt WordNets rein semantische Klassifikation Verbgruppen mit unterschiedlichen syntaktischen Eigenschaften bzw. erfasst nicht immer die syntaktisch regelmäßigen Beziehungen zwischen Unterklassen in separaten Bäumen. ${ }^{2}$

Gross (1975) stellt eine andere semantisch-syntaktische Verbklassifikation dar, die feinkörniger als Levins (1993) ist und wenig Einsicht in die Regelmäßigkeit des Lexikons gewährt. VerbNet (Kipper et al. 2008) stellt einen Versuch dar, WordNet mit Levins Klassifikation auf automatischem Wege miteinander in Einklang zu bringen. Darüber hinaus verfeinert und ergänzt VerbNet die Levinsche Klassifizierung mit dem Ziel, semantische und syntaktische Klassen besser aufeinander abzustimmen. 


\section{FrameNet}

FrameNet (Baker/Fillmore/Cronin 2003, http://framenet.icsi.berkeley.edu/), eine maschinell lesbare lexikalische Ressource für das Englische, basiert auf Fillmores Theorie der Frame-Semantik (Fillmore 1982). Durch die einzelne Wörter übergreifende Perspektive der Darstellung in FrameNet wird die Bedeutung der Wörter nicht isoliert beschrieben, sondern eingebettet in als „Frames" bezeichnete kognitive Schemata - sprachlich beschriebene Situationen oder Kontexte. Der Ansatz von FrameNet basiert auf der Annahme, dass die Bedeutung vieler Wörter am besten im Zusammenhang mit einem Frame sowie dessen Mitspielern und Requisiten - so genannten „FrameElementen" - verstanden werden kann. Der Unterschied zwischen WordNet und FrameNet kann daher grob als paradigmatisch vs. syntagmatisch dargestellt werden.

Lesarten in WordNet entsprechen in FrameNet sog. „Lexical Units“, d.h., Wortformen (Lemmata), die einem bestimmten Frame angehören. Ein Frame wird von einem Nomen, Verb oder Adjektiv evoziert und kann „Lexical Units" aus allen Wortklassen enthalten. Wenn eine Wortform mehrere Frames evoziert, ist sie polysem und wird auf verschiedene „Lexical Units" abgebildet. Zum Beispiel wird das Verb tie up auf Basis der Kontexte in (20) und (21) zwei unterschiedlichen Frames und somit zwei Lesarten zugewiesen:

(20) tie up the deal (Activity-Finish Frame)

(21) tie up the prisoners (Immobilization Frame)

Frame-Elemente ähneln semantischen Rollen und können „Core“- oder „Non-Core"-Status haben; dies entspricht ungefähr dem Unterschied zwischen streng subkategorisierten und optionalen Argumenten (oder Adjunkten) von Verben. Das „Cutting"-Frame zum Beispiel umfasst das Verb cut und die „Core“-Frame-Elemente „Agens", „Item" (die Entität, die (ab)geschnitten oder zerschnitten wird) und „Pieces" (die aus dem Schneiden resultierenden Teile). „Non-Core“-Frame-Elemente sind „Instrument" und „Manner" (die Art und Weise des Schneidens). Letztere müssen nicht sprachlich ausgedrückt werden, obwohl unser Weltwissen sie als notwendige Elemente des Ereignisses voraussetzt.

Wortformen werden einem Frame zugewiesen, wenn „Core“-FrameElemente Wörtern in einer auf Korpusdaten basierenden Konstruktion zugewiesen werden können, und wenn sie dieselbe oder eine ähnliche Situation beschreiben. Grundlegend für den Ansatz von FrameNet ist die Annahme, dass das Sprecherwissen über ein Wort Wissen über dessen Frame-Elemente einschließt, und dass dieses Wissen nicht separat, sondern zusammen mit der lexikalischen Repräsentation des jeweiligen Wortes dargestellt werden soll. 
Ein Beispiel ist der „Claim_ownership“-Frame, den FrameNet folgendermaßen definiert:

(22) A Claimant asserts his or her right to possession of a piece of Property (either real, such as land, or private). The Claimant may be acting on the behalf of a Beneficiary.

Korpusbeispiele, die diesen Frame illustrieren, sind (23)-(25):

(23) The King claims this land and surrounding waters.

(24) Ioping to strike it rich, Thaddeus Gulch staked his claim by the river bed.

(25) I have dibs on the last piece of cake.

Zum „Claim_ownership"-Frame gehören die „Core“-Frame-Elemente "Claimant" (instanziiert durch King, Thaddeus Gulch, I) und "Property" (instanziiert durch this land and surrounding waters, his slaim by the river bed, dibs on the last piece of cakej). Frames werden typischerweise von Verben evoziert, im Fall vom „Claim_ownership"-Frame sind es u.a. die Verben call und claim.

Ein Frame schließt oft Wörter ein, die in WordNet als Synonyme (shut, close), Antonyme (love, hate), Troponyme (walk-stroll), Subevents (buy-pay) kodiert sind. Das bedeutet, dass die Zugehörigkeit zu einem Frame nicht durch syntaktische Eigenschaften wie Subkategorisierung und Argumentalternationen bestimmt wird. Ein Beispiel sind Emotionsverben, von denen sich viele im „Experiencer Subject"-Frame finden, darunter love und hate. Enyy (beneiden') ist ebenfalls diesem Frame zugewiesen, obwohl die syntaktischen Eigenschaften dieses Verbs sich von denen von love und hate unterscheiden: eny hat nicht nur ein zusätzliches obligatorisches FrameElement (die Entität, um die man jemanden beneidet), sondern es erscheint im Gegensatz zu den beiden anderen Verben auch in der Doppelobjektkonstruktion, wie (26) zeigt:

(26) a. I envy you your talent.

b. ${ }^{*}$ I love you your sense of humor.

c. *I hate you your rudeness.

Das derzeitige Bedeutungsinventar von FrameNet ist im Allgemeinen grobkörniger als das von WordNet, was zum Teil darauf zurückzuführen ist, dass FrameNet eine neuere Ressource ist und sich noch im Aufbau befindet. Kleinere Bedeutungsinventare sind vom Standpunkt der maschinellen Sprachverarbeitung, bei der die Disambiguierung von Lesarten eine beachtliche Herausforderung darstellt, wünschenswert. Grobkörnige Unterscheidungen sind jedoch nur dann vorteilhaft, wenn der richtige Grad an Unterspezifikation getroffen wird, so dass alle Lesarten klar unterschieden werden können und sich nicht überlappen. 
Wenn große Verbgruppen wie in FrameNet zusammengeführt werden, ist damit immer die Gefahr verbunden, dass die Möglichkeit feinerer linguistischer Analysen, die wichtige Generalisierungen erlauben, verloren geht. Zu FrameNets "Cutting"-Frame gehören beispielsweise sowohl Verben, die unterschiedliche Arten des Schneidens bezeichnen (carve, chop, mince, pare) als auch denominale Verben, die die durch das Schneideereignis geschaffenen Formen bezeichnen (cube, fillet, dice, slice). In WordNet befinden sich diese beiden Verbklassen in verschiedenen Bäumen mit den Wurzelverben separate bzw. dem Synset \{make, create\}. Die Argumentprojektionen der Verben werden in jedem Baum widergespiegelt: Im Gegensatz zu den Verben, die dem Synset \{separate\} zugeordnet sind, lassen die, die dem Synset $\{$ make, create $\}$ untergeordnet sind, keine Präpositionalphrase zu, in der das „Pieces"-Argument (die aus dem Schneiden resultierenden Teile) wiederholt wird:

(27) The chef carved/minced/chopped the vegetables into pieces/cubes/ slices/dice.

(28) The chef cubed/diced/sliced the vegetables (*into pieces/chunks).

FrameNet verzichtet auch auf die Unterscheidung von Verben mit verwandten aber unterschiedlichen transitiven und intransitiven Lesarten. Dass dadurch eine semantische Unterscheidung verloren gehen mag, zeigt z.B. eine Analyse des Verbs eat (Fellbaum/Kegl 1990). Das intransitive Verb eat weist zwei Lesarten auf, die sich in zwei verschiedenen Unterklassen von Verben widerspiegeln. Troponyme wie munch und nibble gehören zur inergativen Lesart (29). Die zweite intransitive Lesart bezieht ein Argument ein, welches sich auf eine Mahlzeit bezieht; diese Unterklasse schließt die Verben breakfast, lunch, dine und snack ein (30):

(29) The kids are eating/munching/nibbling all day.

(30) Did you eat/breakfast/dine already?

FrameNet kodiert „cross-Frame“-Relationen wie Entailment und Hyperonymie, die den Relationen zwischen Synsets in WordNet ähneln. Der Frame "Crime scenario" hat die Subframes "Committing Crime", "Criminal Investigation" und "Criminal Process". Gemeinsame Frame-Elemente sind Crime and Perpetrator. Weiterhin ist ein „Perspective on"-Frame kodiert, der z.B. die Frames „Commerce_buy" und „Commerce_sell" verbindet: kaufen und verkaufen sind zwei unterschiedliche Perspektiven ein und desselben Ereignisses. 


\section{Verben in WordNet und FrameNet: Das Beispiel play}

Ein gutes Beispiel für die beiden unterschiedlichen Ansätze zur lexikalischsemantischen Analyse ist das Verb play in seiner Bedeutung, Musik spielen (eine hier nicht ausgeführte Analyse des entsprechenden deutschen Verbs spielen fiele wahrscheinlich sehr ähnlich aus). WordNet unterscheidet die in den vier Synsets (31-34) angeführten Lesarten, von denen jede einer anderen Baumstruktur zugeordnet ist:

(31) play an instrument (play the flute) $\Rightarrow$ sound (cause to produce sound)

(32) play a piece of music/kind or piece of music (play Beethoven's Ninth; play Bach; play a waltz) $\Rightarrow$ perform, render

(33) play a location (he's playing Carnegie Hall/New York) $\Rightarrow$ appear

(34) play, make music (the band played all night) $\Rightarrow$ perform

Die Lesarten in (31-33) sind transitiv, die in (34) ist intransitiv (unergativ). Diese Lesarten sind alle verwandt: der Gebrauch von play in einer dieser Lesarten schließt die anderen Lesarten nicht aus. Durch die Verwendung von play mit Bezug auf ein bestimmtes Ereignis kann jede dieser vier Lesarten evoziert werden; jede von ihnen wird aber von einem jeweils anderen Aspekt ein und desselben Ereignisses evoziert. Die Unterscheidung der vier Lesarten von play in WordNet beruht auf der Zuordnung zu unterschiedlichen semantischen Hierarchien, spiegelt sich aber auch in unterschiedlichen syntaktischen Eigenschaften, insbesondere Argumentprojektionen, wider. Erstens können nur das Instrument- und das Ortsargument, aber nicht das Musikstück-Argument der transitiven Lesarten als Adjunkte ausgedrückt werden:

(35) She is still playing on the old piano.

(36) He played in Carnegie Hall/New York.

(37) *She played $\{$ on $/$ in $\}\{$ Bach/a waltz $\}$.

Zweitens kann die Lesart, cause to produce sound ${ }^{c}$ im Gegensatz zu der als ,play a location' paraphrasierten in einer Mittel-Konstruktion erscheinen:

(38) This old piano still plays well.

(39) *Carnegie I Iall plays easily/well.

Drittens können die Instrument- und die Musikstück-Argumente im Gegensatz zum Ortsargument passiviert werden:

(40) The piano/waltz was played at midnight.

(41) *Carnegie Hall was played at midnight. 
Viertens können die Instrument- und die Musikstück/Musik/Komponisten-Argumente im Gegensatz zum Ortsargument als Subjekte des Verbs erscheinen:

(42) The piano played all night.

(43) The waltz/Strauss played all night.

(44) *Carnegie Hall played all night.

Diese Daten scheinen zunächst anzudeuten, dass die durch (31) und (32) exemplifizierten Lesarten (,sound ${ }^{c}$ bzw. ,perform/render) zu einer Lesart zusammengeführt werden könnten. Dagegen spricht aber die Beobachtung, dass die benefaktive Dativ-Konstruktion nur das Musikstück oder den Komponisten, nicht aber das Instrument, als zweites Argument erlaubt:

She played a waltz/some Bach/the oboe for me.

Tabelle 1 fasst die Eigenschaften der verschiedenen Argumente von play mit Bezug auf Alternationen zusammen, die aus WordNets semantisch basierter Klassifikation der drei transitiven Lesarten hervorgehen. Die Tabelle zeigt, dass die auf der Zuordnung zu verschiedenen Uberbegriffen beruhende Erstellung von drei Lesarten syntaktisch gerechtfertigt scheint.

\begin{tabular}{|l|c|c|c|c|c|}
\hline Argument/Syntax & Adjunkt & Mittel & Passiv & Subjekt & Benefaktiv \\
\hline Instrument & + & + & + & + & - \\
\hline Musikstück & - & $?$ & + & + & + \\
\hline Ort & + & - & - & - & - \\
\hline
\end{tabular}

Tab. 1: Eigenschaften der Argumente von play mit Bezug auf Alternationen

In FrameNet ist play mehreren miteinander verlinkten Frames zugeordnet. Jedes Frame ist mit einem deskriptiven Etikett bezeichnet: „Music“, „Instrumental_music" , „Play_instrument ${ }^{c}$, ,Play_tune ${ }^{c}$, „Tune_plays", „Instrument_plays", ,Cause-To_make_sound", „Perform_Location" ${ }^{\prime \prime}$,Core" Frame-Elemente sind „Performer" , „Tune" , „Instrument", „Agent/Cause“, „Sound_maker", „Venue", „Score". Die verschiedenen Frames sind mit je einer Untermenge von Frame-Elementen verbunden, die wiederum durch bestimmte „Lexical Units“ instanziiert werden können. In FrameNet werden die „Lexical Units" nicht syntaktisch beschrieben oder klassifiziert; Frames und die dazugehörenden Frame-Elemente werden intuitiv und semantisch unterschieden.

Die Einträge von FrameNet basieren ausschließlich auf Belegen, die aus dem British National Corpus und dem American National Corpus gewonnen wurden. Da es nicht immer Korpusbelege für alle möglichen Argu- 
mentbelegungen eines gegebenen Verbs gibt, enthält FrameNet für viele Verben, die einem Frame zugeordnet sind, keine Beispiele, und die verschiedenen Valenzen sind somit nicht erschöpfend erläutert. Ebenso werden aufgrund unvollständiger Korpusdaten nicht alle Frame-Elemente, die Argumente oder Adjunkte eines Verbs sein können, in FrameNet mit Beispielen belegt. WordNets hierarchisch angeordnete Lexeme sind hier von Nutzen, da man davon ausgehen kann, dass die meisten Lexeme in einer gegeben Baumstruktur dasselbe Frame-Element instanziieren können.

\section{Abgleichung von WordNet und FrameNet}

Was können die beiden lexikalischen Ressourcen, die das englische Verblexikon großflächig und zum Teil komplementär in Bezug auf die erfassten Eigenschaften abdecken, über semantische und syntaktische Klassen aussagen? Levins (1993) Studie hat die Hypothese, dass syntaktische Eigenschaften von Verben semantisch intuitiv homogene Verbllassen widerspiegeln, größtenteils bestätigt. WordNet klassifiziert und unterscheidet Verben in mehr oder weniger spezifische lexikalisierte Abbildungen von Ereignissen; dieser Ansatz korreliert nur schwach mit einer syntaktischen Klassifikation, vor allem, wenn feinkörnige syntaktische Unterschiede beachtet werden. FrameNet zeigt, dass semantisch ähnliche Verben dieselben oder verwandte Frames und Frame-Elemente evozieren. Trotz einiger Ausnahmen und Unregelmäßigkeiten zeigen die drei verschiedenen Ansätze klare Muster und bestätigen die weitgehende Systematik im Verblexikon. Die unterschiedlichen Klassifikationen können daher nicht als imkompatibel angesehen, sondern sollten vielmehr als komplementär betrachtet werden; jeder Ansatz hebt unterschiedliche Aspekte einzelner Verben und des gesamten Verblexikons hervor. Damit erhebt sich die Frage, ob und wie die umfassende Klassifikation von FrameNet mit der feinkörnigeren von WordNet abgeglichen werden kann.

Ein zweiter potenzieller Gewinn der Abgleichung ist ein besseres Inventar von semantischen Rollen. Textverstehen setzt voraus, dass Satzkonstituenten nicht nur mit syntaktischen Funktionen wie Subjekt und Objekt, sondern darüber hinaus mit semantischen Rollen assoziiert werden können. Beim semantischen Parsing wird versucht, Rollen wie Agens, Patiens, Instrument, Benefizient, Zeit und Ort voneinander zu unterscheiden. Die automatische Abbildung von Satzkonstituenten mit einer größeren Anzahl von semantischen Rollen und Frame-Elementen, die feinkörniger voneinander unterschieden werden, ist eine bisher noch ungelöste Aufgabe. Die Abgleichung von WordNet und FrameNet kann eine optimale Anzahl von semantischen Rollen identifizieren, die zum einen ein tiefes semantisches Verstehen ermöglichen, zum anderen differenziert genug sind, damit ein 
automatisches System lernen kann, sie zu klassifizieren und zu erkennen. Zentral damit verbunden ist die Frage, ob das semantische Rolleninventar auf gewisse Verben und Verbklassen abgestimmt werden sollte.

Die Frame-Elemente von FrameNet können als framespezifische semantische Rollen angesehen werden. Obwohl die Anzahl der Frame-Elemente bereits sehr groß ist und mit fortschreitender Entwicklung der Ressource und der neuen Frames noch wächst, können manche Frame-Elemente in ein unterspezifiziertes verschmolzen werden. Zum Beispiel unterscheiden sich der Rezipient im „Sending"-Frame (47a) und der Benefizient im „Creating"-Frame (47b) nur semantisch aber nicht syntaktisch:

a. I sent Bill a poem.

b. I wrote Bill a poem.

Das Frame-Element „Benefizient“ könnte mit dem Rezipienten verschmolzen werden; beide sind intendierte Empfänger des Themenarguments. Eine kleinere Anzahl von Frame-Elementen erleichtert sowohl die manuelle als auch die automatische Annotation. Umgekehrt können zukünftige Analysen von Frame-Elementen und dazugehörigen Lexemklassen zu feineren Differenzierungen führen.

Ein mit Frame-Elementen annotiertes Korpus kann die Trainings- und Testdaten für das Lernen von semantischem Parsing liefern, und automatische Systeme können verschiedene Granularitäten von Frame-Elementen lernen.

\section{Was kann abgeglichen werden?}

WordNet und FrameNet eignen sich gut für eine Verknüpfung: „Lexical Units $^{c c}$, die in FrameNet bestimmte FrameElemente instanziieren, können mit spezifischen Lesarten (Synsets) in WordNet verlinkt werden. So sollte zum Beispiel das „Core“-Frame-Element „Text" im „Reading"-Frame, für das das „Lexical Unit" paper angegeben ist, nur mit den entsprechenden Lesarten von paper in WordNet, $(48 \mathrm{a}, \mathrm{b})$, annotiert werden, aber nicht mit den Lesarten in $(48 \mathrm{c}, \mathrm{d})$ :
a. \{composition, report, paper, theme (an essay)\}
b. nnewspaper, paper (a daily or weekly publication on folded sheets)\}
c. $\{$ paper (a material made of cellulose pulp)\}
d. \{newspaper, paper, newspaper publisher (a business firm that publishes newspapers)\}

Ein anderes Beispiel ist das polyseme Verb devour (,verschlingen'), das in je einer WordNet-Lesart mit dem „Reading"-Frame und dem „Ingestion" Frame verbunden ist. 
Die Organisation von WordNet erlaubt es, die Abgleichungen automatisch zu erweitern, indem z.B. die Synset-Mitglieder und die Hyperonyme der WordNet-Lesarten, die mit Frame-Elementen verknüpft sind, ebenfalls mit dem entsprechenden Frame-Element verlinkt werden.

\section{Annotation des American National Corpus mit WordNet und FrameNet}

Solange objektive Maßstäbe für ein optimales Bedeutungsinventar fehlen, können Klassifikationen dadurch miteinander verglichen werden, dass derselbe Text mit den unterschiedlichen Bedeutungsinventaren von WordNet und FrameNet annotiert wird. So können nicht nur äquivalente Einträge, sondern auch redundante und obskure oder seltene Lesarten einer polysemen Wortform identifiziert werden. Ein zusätzlicher Gewinn dieser doppelten Annotation besteht darin, dass maschinelle Anwendungen die verschiedenen Informationen, die mit dem jeweiligen Eintrag in WordNet und FrameNet verbunden sind und sich oft gegenseitig bereichern, kombiniert nutzen können.

Ide et al. (2008) annotieren zurzeit ausgewählte Zielwörter (Wortformen) in einem Teil des American National Corpus (uww.americannationalcorpus.org; Ide/Suderman 2004) mit „Lexical Units“ in FrameNet und Synsets in WordNet. Kriterien für die Auswahl der 100 Zielwörter zur Annotation in diesem Manually Annotated Sub-Corpus (MASC) sind Wortklasse, Häufigkeit und Polysemie bzw. Homonymie. Die jeweilige Anzahl der als Zielwörter ausgewählten Nomen, Verben und Adjektive reflektiert grob die Proportionen im gesamten Lexikon (ca. 6:3:1). Da zum einen vermieden werden sollte, dass sich durch dieses Auswahlverfahren eine nicht zu bewältigende Anzahl von Tokens ergeben würde, zum anderen aber auch sichergestellt werden sollte, dass die Annotationen für diverse Anwendungen von maximalem Nutzen sind, werden nur solche Wortformen als Zielwörter ausgewählt, die weder extrem häufig noch extrem selten im Korpus vorkommen. Darüber hinaus wird eine ausgeglichene Mischung von homonymen und polysemen Lesarten angestrebt. Zu den bereits annotierten Wortformen gehören die Nomen nindow, date, part, rate, success, time, work, rule, die Verben lose, win, read, write, serve, try, forget, say, tell und die Adjektive warm, poor, rapid, curious, long, quiet, fair.

Nach der manuellen Bestimmung der Zielwörter annotieren speziell ausgebildete Studenten fünfzig beliebig ausgewählte Tokens pro Zielwort und liefern den Lexikografen detaillierte kritische Kommentare. Dabei werden offensichtliche Fehler, wie doppelte oder fehlende Einträge, entdeckt und verbessert. Die daraufhin revidierten Einträge dienen als Basis für die großflächige Annotation - bis zu 1.000 Tokens pro Zielwort - im Korpus. 
Unser Ziel ist nicht, die Überlegenheit einer lexikalischen Ressource gegenüber der anderen zu beweisen. Vielmehr dienen die Annotationsdaten der Korrektur fehlender oder doppelter Einträge in beiden Ressourcen. Ein weiteres Ziel besteht darin, WordNet und FrameNet so weit wie möglich aufeinander abzustimmen.

Da das Bedeutungsinventar von WordNet eher feinkörnig und das von FrameNet eher grobkörnig ist, ergeben sich viele mehrfach-zu-einfachAbbildungen. Fellbaum/Baker (ersch.) und Baker/Fellbaum (2009) erstellen eine Typologie der Nichtübereinstimmungen. In dem seltenen Fall, dass ein Synset und ein Frame genau dieselben Lexeme enthalten, wird diese Korrespondenz einfach festgehalten werden. Suspicious zum Beispiel hat zwei Bedeutungen in WordNet:

a. \{fishy, funny, shady, suspect, suspicious\}

b. \{leery, mistrustful, suspicious, untrusting, wary\}

Diese beiden Lesarten entsprechen den „Lexical Units" in zwei Frames, „Suspiciousness", ein Subframe von „Social Behavior Evaluation", und „Awareness", ein Subframe von „Mental Activity“.

Häufig sind die Wörter in einem Frame eine Untermenge der Wörter in einem Synset. Dann werden die in WordNet mit dem Zielwort verlinkten Wörter als Synonyme (leery) und Antonyme (trustful) und oft auch als Hyperonyme den entsprechenden Frames als „Lexical Units“ hinzugefügt, was zu einer effizienten Erweiterung von FrameNet führt.

WordNet hat oft mehr Lesarten als FrameNet, insbesondere technische oder seltenere Lesarten. So ist z.B. die Lesart des Adjektivs Long, die mit ,holding securities or commodities in expectation of a rise in prices ' paraphrasiert werden kann, nicht in FrameNet vertreten. Wenn keine entsprechenden Korpusbelege gefunden werden, werden solche Lesarten auch nicht in FrameNet eingegliedert; die Diskrepanz zwischen den Bedeutungsinventaren der beiden Ressourcen scheint hier unproblematisch.

Zwei Synsets, die Untermengen der „Lexical Units“ in einem Frame sind, werden auf eine mögliche Zusammenlegung hin untersucht. Z.B. wurden die zwei Synsets in (50), die einem Frame entsprechen, zusammengeführt; FrameNet kodiert die metaphorische Lesart des Adjektivs legendary nicht gesondert von der wörtlichen.

(50) a. \{legendary (so celebrated as to having taken on the nature of a legend) $\}$

b. $\{$ fabled, legendary (celebrated in fable or legend)\}

Der umgekehrte Fall, dass die „Lexical Units“ zweier Frames Untermengen eines Synsets sind, was die Zusammenlegung der Frames nahelegen würde, ist seltener. Zum Beispiel gibt WordNet nur eine Lesart des Adjektivs successfulan: 
(51) \{successful (having succeeded or being marked by a favorable outcome: ,a successful architect; a successful business venture $\left.{ }^{(c)}\right\}$

FrameNet unterscheidet zwei „Lexical Units“ successful; das eine, das einen Agens impliziert, ist dem Frame ,Successful_action "c zugewiesen; ein zweites, ohne implizierten Agens, gehört zum Frame „Success_or_failure“.

In den meisten Fällen erfolgt die Abgleichung mittels der Über- bzw. Unterordnungsrelation zwischen Synsets, die der ,generalization"-Relation in FrameNet entspricht. So entspricht z.B. die Beziehung zwischen den zwei Frames „Inhibit Movement" und „Imprisonment" dem Troponympaar detain - incarcerate in WordNet, und die "Lexical Units" in dem übergeordneten Frame (,Inhibit_Movement") werden vom untergordneten Frame „Imprisonment" automatisch aufgenommen.

\section{Automatische Abgleichung}

Für viele der ausgewählten Zielwörter befinden sich mehr als 1.000 Tokens - unsere Obergrenze für manuelle Annotation - im Korpus. Automatische Annotation der zusätzlichen Tokens ist geplant; dabei werden die manuellen Tags als Trainings- und Testdaten für den Lernalgorithmus dienen. Verschiedene Versuche zur automatischen Abgleichung wurden bereits unternommen; sie sind zum Teil unbeaufsichtigt und nicht auf manuelle Annotationen gestützt. Wir fassen kurz einige Ansätze zusammen.

Shi/Mihalcea (2005) gleichen Verben in FrameNet und WordNet halbautomatisch ab. Zuerst wurden für 3.000 „Lexical Units“ in FrameNet die entsprechenden WordNet-Lesarten manuell identifiziert. Shi und Mihalcea erweitern die manuelle Abgleichung sodann automatisch und stützen sich dabei auf die Annahme, dass Synonyme und Antonyme eines Verbs aufgrund ihrer in WordNet kodierten semantischen Ähnlichkeit Frame-Elemente im selben Frame sind.

Chow und Websters (2007) Abgleichung erfolgt indirekt über die Suggested Upper Merged Ontology (SUMO), eine formale Ontologie, die manuell auf WordNet abgebildet wurde (Niles/Pease 2003). Ausgangspunkt für die Abgleichungen von Chow und Webster sind die Abgleichungen von Shi/Mihalcea (2005) von „Lexical Units“ in Frames mit Wörtern in einem Synset. Diesen Abgleichungen fügen Chow/Webster alle Synset-Mitglieder sowie alle in WordNet verlinkten Wörter hinzu. Diese Menge von Wörtern ergibt eine semantische Domäne unter der Annahme, dass die in WordNet miteinander verknüpften Wörter ein und derselben semantischen Domäne angehören.

Zum Beispiel werden die WordNet Synsets in (52) der Domäne, ,Statement" zugewiesen, da sie alle in WordNet mit dem Verb address in Synset (53) vernetzt sind und dieses Verb manuell von Shi/Mihalcea auf die entsprechende „Lexical Unit“ abgebildet wurde: 
(52) \{communicate, intercommunicate\}

\{betray\}

\{inform

\{disclaim\}

\{address, turn to

Um die Abgleichung so fehlerfrei wie möglich zu gestalten, beziehen Chow/ Webster sich auf Domänen in SUMO und die unabhängig durchgeführten SUMO-WordNet-Abgleichungen (Niles/Pease 2003).

Wie andere Abgleichungsansätze basiert der von Chow/Webster auf der nicht immer korrekten Annahme, dass in WordNet verlinkte Lesarten den „Lexical Units" in einem gegebenen Frame entsprechen. Diese Annahme trifft zwar relativ systematisch für alle Mitglieder eines Synsets zu, ist aber weniger verlässlich für andere semantische Relationen. Zum Beispiel sind die Verben cook und heat in WordNet durch Entailment verbunden. Cook ist ein Kreationsverb, aber beat drückt eine Zustandsveränderung aus. Es folgt also nicht zwingend, dass die in WordNet vernetzten Lesarten dieser Verben derselben Domäne angehören und dass sie die entsprechenden semantisch verwandten Lesarten der „Lexical Units" in einem Frame sind.

Ein weiteres Problem mit Chows und Websters Ansatz ist, dass Frames sehr feinkörnig sind, und die in WordNet verwandten Verben in fast allen Fällen nicht nur einem, sondern einer Anzahl von Frames zugewiesen werden müssen. Word Nets Relationen sind nicht dazu geeignet, diese feinkörnigen Zuteilungen automatisch zu erstellen.

Schließlich sind die Zuteilungen zu Domänen in WordNet oft arbiträr und beruhen nicht auf semantischer Ähnlichkeit; diese störenden Ungenauigkeiten sind auch in der Abgleichung mit den Domänen von SUMO übernommen.

Das Ziel von Burchardt/Erk/Frank (2005) ist, die Anzahl der „Lexical Units“" in FrameNet zu erhöhen. Die Methode, ein so genannter „Umweg“ über WordNet, macht Gebrauch von den Relationen in WordNet und erreicht gleichzeitig eine Abgleichung der beiden Ressourcen. In einem ersten Schritt werden ausgewählte Zielwörter mittels der auf WordNets Ähnlichkeitsmaßen basierten Methode von Banerjee/Pedersen (2003) disambiguiert. Diese Maße beruhen auf der Annahme, dass diejenigen Lesarten polysemer Wörter, die in WordNet miteinander verlinkt sind und sich in gegenseitiger „Nähe“ im Netz befinden, semantisch ähnlich sind; Relationen können dabei unterschiedlich gewichtet werden. Burchardt/Erk/ Frank (2005) fügen sodann alle in WordNet verlinkten Wörter den disambiguierten Zielwörtern hinzu (Hyperonyme, Troponyme, Antonyme, Meronyme, etc.). Der Frame, in dem ein Großteil dieser Wörter als „Lexical Units ${ }^{\text {c }}$ vorkommt, wird identifiziert, und das Zielwort wird ihm zu- 
gewiesen. Multiple Kandidaten-Frames werden nach einem Gewichtsschema evaluiert. Ein einfaches Beispiel ist das Adjektiv new, welches aufgrund der Antonymierelation zu old dem ,Age“-Frame zugewiesen werden konnte.

Wie Chow/Webster stützen sich Burchardt/Erk/Frank (2005) auf die nicht immer verlässliche Annahme, dass die semantische Ähnlichkeit von Wörtern in WordNet mit gemeinsamer Frame-Zugehörigkeit in FrameNet korrespondiert. Oltramari (2006) verfeinert die Methode von Burchardt/ Erk/Frank (2005) mit einer statistischen Analyse der Verteilung der Frames unter WordNets Hyponymen; dadurch werden die rein semantischen Ähnlichkeitsmaße durch ein quantitatives $\mathrm{Maß}$ ergänzt.

Eine Reihe anderer Ansätze zur automatischen Abgleichung von Lesarten in WordNet und „Lexical Units" in FrameNet verlassen sich nicht nur auf die in WordNet kodierten Relationen, sondern ziehen zusätzlich die Wörter, die in den Definitionen der Synsets vorkommen, in Betracht. Tonelli/Pianta (2009) gleichen die „Lexical Units" von FrameNet mit den Synsets von WordNet ab, indem sie die Anzahl der gemeinsamen Wortformen in der Definition eines WordNet-Synsets und der korrespondierenden lexikalischen Einheit in FrameNet messen. Ferrández et al. (2010) verfeinern diese Methode und erzielen etwas genauere Resultate, indem sie die Ähnlichkeit der lexikalischen Umgebung von Paaren von „Lexical Units“ in FrameNet und Synsets in WordNet messen.

Ides (2006) Abgleichung geht in die umgekehrte Richtung, von den „Lexical Units“ in FrameNet zu den Synset-Mitgliedern in WordNet. Ide disambiguiert je zwei „Lexical Units" in einem Frame, indem sie die Ähnlichkeitsmaße für die Disambiguierung von Patwardhan/Pedersen (2006) anwendet. Diese Maße berechnen und gewichten die semantische Ähnlichkeit zweier Wortformen, indem sie alle Lesarten dieser Wortformen untersuchen und ein Ranking erstellen. Die Maße beruhen wie die von Banerjee/ Pedersen (2003) auf WordNets Struktur (diversen WordNet-Relationen und lexikalischer Überlappung der „Lexical Units“ mit Lemmata in den Definitionen und Beispielsätzen). Ides Grundannahme dabei ist, dass zwei Wortformen, die beide einem Frame angehören, semantisch verwandt sind, und dass diese Verwandtschaft in WordNet erschlossen werden kann. Die Lesarten der „Lexical Units“, die von Patwardhan/Pedersens (2006) Ähnlichkeitsmaßen am höchsten gerankt werden, werden als die für den Frame zutreffenden angesehen.

Ein Beispiel sind die Wortformen cast und pitch, die beide zum „Cause Motion"-Frame gehören, aber mehrfache Lesarten haben, welche anderen Frames (einschließlich „Create Physical Artwork ${ }^{c 6}$ und „Encoding ${ }^{6 \text { ) }}$ zugewiesen sind. Patwardhan und Pedersens (2006) Ähnlichkeitsmaße disambiguieren dieses Wortpaar und identifizieren die in WordNet verwandten „Cause Motion“-Lesarten in WordNet. 
Automatische Abgleichungen können manuelle Annotation nicht ersetzen. Sie beruhen auf Ressourcen wie WordNet, die nicht für Abgleichungszwecke konstruiert wurden und deren interne Verknüpfung verschiedene Arten und Grade von semantischer Ähnlichkeit kodieren, die nicht unbedingt der Ähnlichkeit von „Lexical Units“ in einem Frame entsprechen. Wir erwarten, dass das Vorgehen von Baker/Fellbaum (2009) und Fellbaum/ Baker (ersch.), das die Abgleichung von WordNet und FrameNet gezielter mittels manueller Annotation von WordNet-Lesarten und FrameNet-,Lexical Units" vornimmt, verlässlichere Daten als Input für eine umfangreiche automatische Abgleichung liefert.

\section{Annotation eines deutschen Korpus mit Frames: SALSA}

Das SALSA Projekt (Saarbruecken Lexical Semantics Annotation and Analysis; Burchardt et al. 2009) hat sich, ähnlich wie MASC, zum Ziel gesetzt, ein großes deutsches Korpus mit Frames und Frame-Elementen zu annotieren, auf Grund der Annotationen ein framebasiertes Lexikon zu generieren und schließlich ein datenbasiertes Modell für die automatische Frame-Analyse zu entwickeln. Das Projekt geht von der Annahme aus, dass Frames und Frame-Elemente universalen Status haben. Es ist intuitiv einleuchtend, dass zum Beispiel ein Transfer-Ereignis folgende Argumente hat: Ursprung, Ziel und Thema (die überreichte Entität), unabhängig davon, wie diese FrameElemente syntaktisch und morphologisch realisiert werden. Bisher konnte gezeigt werden, dass sich Frames, die für das Englische entwickelt wurden, gut auf das Deutsche übertragen lassen. Boas (2009) diskutiert das Potenzial sowie die Grenzen der Anwendung von Frames als sprachunabhängige Repräsentationen für übereinzelsprachliche lexikalische Ressourcen.

Systematische Unterschiede zwischen entsprechenden deutschen und englischen Frames sind größtenteils auf sprachspezifische lexikalische und morphosyntaktische Eigenschaften zurückzuführen. So fordert z.B. der Gebrauch des Dativs im Deutschen bei untrennbarem Besitz ein zusätzliches strukturelles Argument, das im Englischen nicht als Nomen oder Pronomen erscheint:

a. Hans hat sich den Arm gebrochen.

b. Hans broke his arm.

a. Der Dieb hat mir die I Iandtasche gestohlen.

b. The thief stole my handbag.

Die deutschen Dativpronomen und die entsprechenden englischen Possessiva können mit einem „Extra-Frame“-Element „Possessor c ${ }^{c}$ etikettiert werden (Burchardt et al. 2009). Eine einheitliche Etikettierung mit einem englischen Aquivalent kann nicht für den so genannten ,ethischen“ Dativ vorgenommen werden, der im Englischen nicht existiert: 
(56) a. Der Patient ist mir gestorben.

b. The patient died $\varnothing$.

In diesen Fällen müsste ein zusätzliches Frame-Element für die deutschen Frames angenommen werden oder - alternativ - das Argument ohne Bezeichnung bleiben. Da die Bedeutung dieses Elements unterspezifiziert und kontextspezifisch ist, wäre die erste Lösung problematisch und würde mehrere Frame-Elemente erfordern. Der ethische Dativ trägt nur wenig zur Satzbedeutung bei und verändert den Wahrheitswert des Satzes nicht. Es wäre schwer, zu argumentieren, dass ein entsprechendes Frame-Element im Englischen existiert, aber nicht auf der Satzoberfläche realisiert wird.

Ein anderer, recht systematischer Unterschied ist, dass Substantivableitungen von Verben im Englischen höchst produktiv, aber im Deutschen weniger häufig sind. Viele englische Verben wie pencil, bookmark und carpet enthalten ein Frame-Element (,Instrument ${ }^{c c}$, ,Material ${ }^{c c}$, usw.), das im Deutschen als eigenständiges Argument und Frame-Element des Verbs ausgedrückt wird.

Die in den SALSA-Frames bestimmten ,Lexical Units" werden nicht auf eine unabhängige Ressource wie das deutsche WordNet (GermaNet) abgebildet. Interessant ist der von Burchardt et al. (2009) durchgeführte Vergleich der durchschnittlichen Anzahl der „Lexical Units “ für eine Wortform mit der durchschnittlichen Anzahl der Lesarten in GermaNet: Letztere ist höher, d.h., die Frame-Analyse ergibt ähnlich wie bei den korrespondierenden englischen Ressourcen durchschnittlich mehr Lesarten pro Wortform.

\section{Schlussfolgerung}

WordNet und FrameNet stellen unterschiedliche Ansätze zur Bestimmung von Lesarten polysemer Wortformen und zur Erschließung des Lexikons dar. Beide sind semantisch ausgerichtet, lassen jedoch Regelmäßigkeiten erkennen, die mit einem syntaktischen Ansatz wie Levins Klassifikation des Verblexikons (1993) kompatibel sind. Eine Abgleichung von WordNet und FrameNet im Rahmen einer umfangreichen Korpusannotation zeigt klar, dass beide Ressourcen überlappende Lesarteninventare haben und miteinander in Einklang gebracht werden können. Einander entsprechende Lesarten zeigen die Ubereinstimmung von Argumentstrukturen und Alternationen von Verben in WordNet mit Frame-Elementen in FrameNet. 


\section{Literatur}

Anderson, Stephen (1971): On the role of deep structure in semantic interpretation. In: Foundations of Language 7, S. 387-396.

Baker, Collin F./Fellbaum, Christiane (2009): WordNet and FrameNet as complementary resources for annotation. In: Association for Computational Linguistics ( $\mathrm{Hg}$ ): Proceedings of the Third Linguistic Annotation Workshop. Singapur, S. 125-129.

Baker, Collin F./Fillmore, Charles J./Cronin, Beau (2003): The structure of the Framenet database. In: International Journal of Lexicography 16, 3, S. 281-296.

Banerjee, Satanjeev/Pedersen, Ted (2003): Extended gloss overlaps as a measure of semantic relatedness. Proceedings in the International Joint Conference on Artificial Intelligence (IJCAI). Acapulco, S. 805-810.

Boas, Hans C. (2009): Semantic frames as interlingual representations for multilingual lexical databases. In: Boas (Hg), S. 59-100.

Boas, Hans C. (Hg.) (2009): Multilingual FrameNets in Computational Lexicography: Methods and applications. (= Trends in Linguistics: Studies and Monographs 200). Berlin.

Boyd-Graber, Jordan et al. (2006): Adding dense, weighted connections to WordNet. In: Sojka, Peter et al. $(\mathrm{Hg})$ : Proceedings of the Third Global WordNet Conference. Jeju Island, Republik Korea, S. 29-35.

Burchardt Aljoscha/Erk, Katrin/Frank, Anette (2005): A WordNet Detour to FrameNet. In: Fisseni, Bernhard et al. (Hg): Sprachtechnologie, mobile Kommunikation und linguistische Ressourcen: Beiträge zur GLDV-Tagung 2005 in Bonn. (= Computer Studies in Langauge and Speech 8). Frankfurt a.M., S. 408-421.

Burchardt, Aljoscha/Erk, Katrin/Frank, Anette et al. (2009): Using FrameNet for the semantic analysis of German: Annotation, representation and automation. In: Boas (Hg.), S. 209-244.

Chow, Ian/Webster, Jonathan (2007): Integration of linguistic resources for verb classification: FrameNet frame, WordNet verb and suggested upper merged ontology. In: Gelbukh, Alexander ( $\mathrm{Hg}$.): Proceedings of the $8^{\text {th }}$ Conference on Computational Linguistics and Intelligent Text Processing (CICLing 2007). Mexico City, S. $1-11$.

Collins, Allan M./Quillian, M. Ross (1969): Retrieval time from Semantic Memory. In: Journal of Verbal Learning and Verbal Behavior 8, S. 240-247.

Cruse, Alan D. (1986): Lexical semantics. Cambridge.

Fagan, Sarah (1992): The syntax and semantics of middle constructions: A study with special reference to German. (= Cambridge Studies in Linguistics 60). Cambridge.

Fellbaum, Christiane (1990): The English verb lexicon as a semantic net. In: International Journal of Lexicography 3, 4, S. 278-301.

Fellbaum, Christiane (Hg.) (1998): WordNet: An electronic lexical database. Cambridge, MA. 
Fellbaum, Christiane (2002): The semantics of troponymy. In: Green, Rebecca/Bean, Carol/Myaeng, Sung Hyon (Hg.): The semantics of relationships: An interdisciplinary perspective. (= Information Science and Knowledge Management 3). Dordrecht, S. 23-34.

Fellbaum, Christiane/Baker, Collin F. (ersch.): Aligning verbs in WordNet and FrameNet. In: Linguistics.

Fellbaum, Christiane/Chaffin, Roger (1990): Some principles of the organization of the verb lexicon. In: Proceedings of the $12^{\text {th }}$ Annual Conference of the Cognitive Science Society. Hillsdale, NJ, S. 420-428.

Fellbaum, Christiane/Grabowski, Joachim/Landes, Shari (1997): Analysis of a handtagging task. In: Association for Computational Linguistics (Hg.): Proceedings of the ACL/Siglex workshop. Washington, DC, S. $34-40$.

Fellbaum, Christiane/Kegl, Judy (1990): Taxonomic structure and object deletion in the English verbal system. In: deJong, Ken/No, Yongkyoon ( $\mathrm{Hg}$.): Proceedings of the Sixth Eastern States Conference on Linguistics. Columbus, OH, S. 94-103.

Fellbaum, Christiane/Zribi-Hertz, Anne (1990): La construction moyenne en français et en anglais: Etude de syntaxe et de sémantique comparée. In: Recherches Linguistiques à Vincennes 18, S. 19-57.

Ferrández, Óscar et al. (2010): Aligning FrameNet and WordNet based on semantic neighborhoods. In: Calzolari, Nicoletta et al. (Hg.): Proceedings of the Seventh Conference on Language Resources and Evaluation (LREC 10). Valetta, S. 310-314.

Fillmore, Charles J. (1982): Frame semantics. In: Linguistic Society of Korea (Hg): Linguistics in the morning calm. Seoul, S. 111-137.

Green, Georgia (1974): Semantics and syntactic regularity. Bloomington, IN.

Gross, Maurice (1975): Méthodes en syntaxe: Régime des constructions complétives. (= Actualités Scientifiques et Industrielles 1365). Paris.

Ide, Nancy (2006): Making senses: Bootstrapping sense-tagged lists of semantically related words. In: Gelbukh, Alexander (Hg:): Computational Linguistics and Intelligent Text, Lecture Notes in Computer Science Vol. 3878. Heidelberg, S. 13-27.

Ide, Nancy/Suderman, Keith (2004): The American National Corpus first release. In: Proceedings of the Fourth Language Resources and Evalation Conference (LREC). Lissabon, S. 1681-1684.

Ide, Nancy et al. (2008): MASC: A Manually Annotated Subcorpus of American English. In: Proceedings of the Sixth Language Resources and Evaluation Conference (LREC). Marrakesch, S. 617-620.

Keyser, Sam J./Roeper, Thomas (1984): On the middle and ergative construction in English. In: Linguistic Inquiry 15, S. 381-416.

Kipper, Karin et al. (2008): A large-scale classification of English verbs. In: Language Resources and Evaluation 42, S. 21-40.

Kunze, Claudia (2000): Extension and use of GermaNet, a lexical-semantic database. In: Proceedings of the Second International Conference on Language Resources and Evaluation. Athen, S. 999-1002. 
Levin, Beth (1993): English verb classes and alternations: A preliminary analysis. Chicago u.a.

Miller, George A./Beckwith, Richard/Fellbaum, Christiane et al. (1990): Introduction to WordNet: An on-line lexical database. In: International Journal of Lexicography 3, 4, S. 235-244.

Nikolova, Sonja/Boyd-Graber, Jordan/Fellbaum, Christiane (ersch.): Collecting semantic similarity ratings to connect concepts in assistive communication tools. In: Mehler, Alexander et al. ( $\mathrm{Hg}$.): Modeling, learning and processing of text technological data structures. Studies in computational intelligence. Heidelberg/New York.

Niles, Ian/Pease, Adam (2003): Linking lexicons and ontologies: Mapping WordNet to the suggested upper merged ontology. In: Arabnia, Hamid (Hg.): Proceedings of the 2003 International Conference on Information and Knowledge Engineering. Las Vegas, S. 412-416.

Oltramari, Alessandro (2006): LexiPass methodology: A conceptual path from frames to senses and back. In: Proceedings of the Fifth International Conference on Lexical Resources in the European Community (LREC). Genua, S. 2311-2314.

Pantel, Patrick/Lin, Dekang (2002): Discovering word senses from text. In: Proceedings of ACM Conference on Knowledge Discovery and Data Mining (KDD-02). Edmonton, S. 613-619.

Patwardhan, Siddhartha/Pedersen, Ted (2006): Using WordNet-based context vectors to estimate the semantic relatedness of concepts. In: Proceedings of the European Association of Computational Linguistics (EACL) 2006 Workshop Making Sense of Sense-Bringing Computational Linguistics and Psycholinguistics Together. Trient, S. $1-8$.

Schulte im Walde, Sabine (ersch.): The induction of verb frames and verb classes from corpora. In: Lüdeling, Anke/Kytö, Merja (Hg.): Corpus linguistics: An international handbook. Berlin.

Shi, Lei/Mihalcea, Rada (2005): Putting pieces together: Combining FrameNet, VerbNet and WordNet for robust semantic parsing. In: Gelbukh, Alexander (Hg.): Proceedings of the Sixth International Conference on Intelligent Text Processing and Computational Linguistics. Mexico City, S. 100-111.

Tonelli, Sara/Pianta, Emanuele (2009): A novel approach to mapping FrameNet lexical units to WordNet synsets. In: Proceedings of Eighth International Conference on Computational Semantics (IWCS-8). Tilburg, S. 342-345.

Woicjik, Richard (1976): Where do instrumental NPs come from? In: Shibatani, Masayoshi $(\mathrm{Hg}$ ): Syntax and semantics 6: The grammar of causative constructions. New York, S. 165-180. 